\title{
O INSTITUTO DE ESTUDOS AVANÇADOS DA UFMG E SUA CONTEXTUALIZAÇÃO COMO UM IEA COM CARACTERÍSTICAS PRÓPRIAS
}

THE INSTITUTE OF ADVANCED TRANSDISCIPLINARY STUDIES OF UFMG AND ITS CONTEXTUALIZATION AS AN INSTITUTE OF ADVANCED STUDIES (IAS) WITH PROPER CHARACTERISTICS

\section{EL INSTITUTO DE ESTUDIOS AVANZADOS DE LA UFMG Y SU CONTEXTUALIZACIÓN COMO UN IEA CON CARACTERÍSTICAS PROPIAS}

\author{
Estevam Barbosa de Las Casas* \\ Aretusa Kelly Alves Duarte*
}

\begin{abstract}
RESUMO
Neste trabalho, recupera-se a história da criação e do desenvolvimento dos Institutos de Estudos Avançados no mundo. Após essa retomada, o trabalho se detém na apresentação do Instituto de Estudos Avançados Transdisciplinares da Universidade Federal de Minas Gerais (IEAT/UFMG), que, como o nome indica, traz a transdisciplinaridade como horizonte principal, no contexto de uma instituição universitária, para a tentativa de lidar com a complexidade dos problemas reais da sociedade. Dessa maneira, apresentam-se algumas iniciativas do IEAT em associar pesquisa de alto nível com aplicação na solução de problemas, utilizando-se metodologias transdisciplinares.
\end{abstract}

Palavras-chave: Instituto de Estudos Avançados. Universidade. Metodologias. Transdisciplinaridade.

\begin{abstract}
In this work, we recover the history of creation and development of the Institutes of Advanced Studies around the world. After such resumption, the text focus on the Institute of Advanced Transdisciplinary Studies of UFMG which, as indicated by its own name, brings up transdisciplinarity as a main horizon in the context of a university institution, for the attempt of dealing with complexity of life-world societal problems. Thus, we present some initiatives of the Institute related to the association between state-

\footnotetext{
*Pós-doutor pela Universidade Politécnica da Catalunha, doutor pela Purdue University, mestre pela Pontifícia Universidade Católica do Rio de Janeiro (PUC Rio) e graduado em Engenharia Civil pela Universidade Federal de Minas Gerais (UFMG); diretor do Instituto de Estudos Avançados Transdisciplinares da UFMG (IEAT/UFMG), professor titular no Departamento de Engenharia de Estruturas da Universidade Federal de Minas Gerais (UFMG). Bolsista de produtividade em pesquisa do CNPq - Nível 1C. E-mail: estevam.lascasas@gmail.com

${ }^{* *}$ Mestra em Administração pela Fundação Dr. Pedro Leopoldo, pós-graduada em Gestão Educacional pela Faculdade Senac Minas, graduada em Letras pela UFMG, assessora acadêmica do EAT/UFMG. E-mail: info@ieat.ufmg.br.
} 
of-the-art research and its application in the solution of problems, using transdisciplinary methodologies.

Keywords: Institute of Advanced Studies. Universities. Methodologies. Transdisciplinarity.

\section{RESUMEN}

En este trabajo, se recupera la historia de la creación y el desarrollo de los Institutos de Estudios Avanzados en el mundo. Después de esa reanudación, el trabajo se detiene en la presentación del Instituto de Estudios Avanzados Transdisciplinares de la Universidad Federal de Minas Gerais, que, como su nombre indica, trae la transdisciplinariedad como horizonte principal, en el contexto de una institución universitaria, para el intento de lidiar con la complejidad de los problemas reales de la sociedad. De esta manera, se presentan algunas iniciativas del Instituto en asociar investigación de alto nivel con aplicación en la solución de problemas, utilizando metodologías transdisciplinares.

Palabras clave: Instituto de Estudios Avanzados. Universidades. Metodologías. Transdisciplinariedad.

\section{INTRODUÇÃO}

$\mathrm{O}$ primeiro Instituto de Estudos Avançados (IEA) foi criado em Princeton, em 1930, e se inspirava no Collège de France e no All Souls College, em Oxford, inclusive quanto à busca por um ambiente que favorecesse a camaradagem, contemplação e tranquilidade. Com um quadro permanente de pesquisadores e "fellows", serviu de exemplo para várias outras iniciativas, nos Estados Unidos e Europa inicialmente, e por lá passaram pesquisadores como Kurt Godel, Albert Einstein e John von Neumann.

O objetivo de um IEA, a se seguir o primeiro deles a ser criado, é "reunir um grupo de cientistas e professores que com seus alunos e assistentes possam devotar-se à tarefa de avançar os limites atuais do conhecimento humano e treinar aqueles que podem 'seguir' neste sentido" (Institute for Advanced Study, 2019, tradução nossa). De acordo com seu fundador e primeiro diretor, Abraham Flexner, seu sonho era criar "Um instituto devotado à pesquisa sem restriçôes na fronteira do conhecimento" (Netherlands Institute for Advanced Study, 2019, tradução nossa). Flexner (1939) considerava que:

Instituiçôes de ensino devem ser devotadas ao cultivo da curiosidade e quanto menos elas são desviadas por considerações a respeito da imediaticidade da aplicação, mais provável 
é que elas contribuam não apenas ao bem-estar humano, mas também à igualmente importante satisfação do interesse intelectual que se pode de fato dizer ter se tornado a paixão dominante da vida intelectual dos tempos modernos.

O segundo IEA a ser criado foi o Dublin Institute for Advanced Studies (DIAS), na Irlanda, em 1940. Assim como o primeiro, este também não tinha ligaçôes administrativas com uma universidade, e dedica-se hoje ao estudo focado na Física Teórica e Cósmica, além de Estudos Celtas. Por iniciativa da Fundação Ford, o terceiro IEA foi criado em Palo Alto, em 1954, sob o nome de Center for Advanced Study in the Behavioral Sciences (CASBS). Com uma estrutura menor, focado em Ciências Humanas e Sociais, o Centro era baseado em fellows, residentes com permanência temporária que, ao contrário de Princeton, não eram selecionados por meio de editais e sim convidados pelo CASBS. Desde 2008, o CASBS passou a integrar a Universidade de Stanford.

Dezesseis anos mais tarde, em 1970, dois IEA foram criados na Europa, um na Holanda, outro na Alemanha. Enquanto o primeiro, o Netherlands Institute for Advanced Study (NIAS), localizado inicialmente em Wassenaar, seguia de perto o modelo de Princeton, em Bielefeld, o Zentrum für Interdisziplinäre Forschung (ZIF), ou Centro para Pesquisa Interdisciplinar, inovava ao ser estruturado e montado juntamente com a Universidade de Bielefeld. Ambos os institutos davam ênfase ao apoio a grupos de pesquisa temática, em vez do trabalho individual priorizado pelos primeiros IEA. A concepção da Universidade de Bielefeld como um centro de pesquisa interdisciplinar, a partir da proposta de Helmut Schelsky, com todas as áreas convivendo em espaços compartilhados, incluía o ZIF não como um anexo ou complemento, mas como uma estrutura definidora de propostas para a pesquisa na Universidade. A proposta do ZIF, de um IEA vinculado a uma universidade, mostrou-se atraente para um grande número de instituições. Uma referência para a história da evolução dos IEA internacionais foi elaborada por Wittrock (2019), diretor do Swedish Collegium for Advanced Study in the Social Sciences, de onde boa parte destes dados foram coletados.

A partir de uma iniciativa do Freiburg Institute for Advanced Studies (FRIAS), foi criada em 2010 a rede University-Based Institutes for Advanced Study (UBIAS), cujo propósito inicial era reunir IEA sediados em universidades, mas que atualmente interliga institutos avançados dos cinco continentes. Até o momento, apenas dois IEA brasileiros fazem parte da rede UBIAS: o IEA da Universidade de São Paulo (USP), desde 2010; e o Instituto de Estudos Avançados Transdisciplinares da Universidade Federal de Minas Gerais (UFMG), filiado em 2018. 
O IEA da USP, criado em 1986, foi o primeiro do gênero na América Latina. Seu objetivo explícito era não concorrer com as estruturas existentes de pesquisa da universidade, mas ativar um espaço de reflexão onde se cultivassem os estudos avançados conduzidos por mestres de excelência nacional e internacional, no interior da instituição. O ano de 1995 marcou a criação do Instituto LatinoAmericano de Estudos Avançados (ILEA) da Universidade Federal do Rio Grande do Sul (UFRGS).

Em 1999, foi criado experimentalmente o Instituto de Estudos Avançados Transdisciplinares (IEAT) da UFMG. De 1999 a 2005, funcionou de forma experimental. Seus propósitos foram definidos na Resolução que o criou:

O IEAT tem como objetivo criar, no âmbito da Universidade Federal de Minas Gerais, um ambiente propício à realização de estudos transdisciplinares, com características de excelência, de ponta e de indução, abrangendo as diversas áreas do conhecimento artísticas, tecnológicas, biológicas, exatas e da terra, humanas e sociais - em seus diferentes âmbitos, índoles e aspectos (Resolução no 08/1999).

Quanto ao caráter que norteava os primeiros IEA, privilegiava-se a pesquisa reflexiva, individual e "pura", com a implicação de que a pesquisa pura é o motor que propulsiona o desenvolvimento tecnológico. Propunha-se a ideia de que a pesquisa devia, necessariamente, descolar-se da busca por aplicaçóes, que seriam uma derivação dessa etapa anterior.

Flexner, em seu ensaio, conforme relata Goddard (2011), propagou que os avanços nos conhecimentos de maior valor prático não provêm de pesquisas baseadas em objetivos, mas daquelas motivadas por curiosidade intelectual. Stokes (1997), em seu influente livro Pasteur's quadrant: basic science and technological innovation, defende que esses caminhos não são necessariamente seguidos. Mostra que a conexão entre pesquisa básica e aplicada pode não existir, ou não ser determinante, ou ainda se dar no sentido inverso, da tecnologia para a inovação científica. E propóe a classificação de um terceiro tipo de pesquisa, em que se avança com as bases teóricas e básicas da ciência simultaneamente com resultados de aplicações imediatas, dando como exemplo o trabalho de Pasteur.

Em se tratando do IEAT, a transdisciplinaridade tem sido uma das bases do Instituto. Nesse sentido, o IEAT já organizou dois seminários sobre educação superior com uma série de encontros, sempre combinando os temas com a necessidade de um enfoque menos departamental e corporativista. Vem também buscando, sempre que possível, coorganizar suas atividades com outras estruturas da UFMG, tais como cursos de pós-graduação, estruturas da Reitoria (como as pró-reitorias acadêmicas) e outros órgãos, como a Diretoria de Relações 
Internacionais, o GIZ - Diretoria de Inovação e Metodologias de Ensino, o Centro de Apoio à Educação a Distância (CAED), laboratórios institucionais e a Coordenadoria de Transferência e Inovação Tecnológica (CTIT). Já em 1999, Brewer refletia:

Universidades e outras instituições de ensino são fontes óbvias de criatividade e inovação, e, na verdade, elas forneceram algo de ambos elementos. Preocupaçōes importantes centramse na pequena quantidade e no ritmo lento dessas, como refletido nas enormes e não resolvidas dificuldades de atingir um desenvolvimento sustentável, gestão do ecossistema, avaliaçôes integradas de sistemas e processos bio-geo-químicos extremamente complexos e clareza sobre intervençôes intencionais humanas em escalas global e regional, bem como local (p. 328).

Diante da dificuldade das estruturas das universidades em lidar com os problemas reais da sociedade moderna, com a complexidade a eles inerente, Brewer (1999) comenta: "O mundo tem problemas, mas as universidades têm departamentos” (p. 328).

Pohl e Hirsch (2007), em proposta elaborada pela Academia Suíça de Pesquisa, propõem que métodos de pesquisa transdisciplinar têm um papel fundamental na solução de problemas do mundo real, frisando a necessidade de participação de atores envolvidos diretamente nos problemas para a busca de uma solução.

Há uma necessidade da pesquisa transdisciplinar quando o conhecimento sobre um problema social relevante é incerto, quando a natureza concreta dos problemas é disputada e quando há muito em jogo para aqueles concernidos pelos problemas e envolvidos em lidar com eles. A pesquisa transdisciplinar lida com campos problemáticos de tal maneira que pode: a) alcançar a complexidade dos problemas; b) levar em conta a diversidade dos problemas reais e as percepçôes científicas dos problemas; c) conectar conhecimento abstrato e caso específico; d) constituir conhecimento e práticas que promovem o que é percebido como sendo o bem comum (Pohl \& Hirsch, 2007, p. 20, tradução nossa).

Conforme tratado por Van Breda e Swilling (2018) em The guiding logics and principles for designing emergent transdisciplinary research processes: learning experiences and reflections from a transdisciplinary urban case study in Enkanini informal settlement, South Africa: "A premissa deste artigo é que as metodologias da pesquisa transdisciplinar desenhadas para contextos do mundo desenvolvido não podem meramente ser replicadas e transferidas para contextos do mundo em desenvolvimento". Diferentes realidades em termos de demandas sociais, pressões e recursos tornam a missão e as metodologias dos IEA, em países em desenvolvimento, bastante particulares. Seminários sobre problemas da atualidade como a exploração do pré-sal, as ameaças às liberdades de expressão e a utilização das jazidas de lítio no Estado de Minas Gerais foram organizados 
pelo IEAT entre 2017 e 2019, com boa participação da comunidade.

Algumas das iniciativas do IEAT baseiam-se diretamente na tentativa de associar pesquisa de alto nível com aplicação na solução de problemas reais (life-world) utilizando-se metodologias transdisciplinares. Nesse sentido, foram selecionados dois grupos de pesquisa em 2017 com propostas fundadas nessas premissas, e mais dois, em 2019, entre eles o Grupo de Pesquisas "Curso de vida e trajetória delinquencial: um estudo exploratório dos eventos e narrativas de jovens em situação de vulnerabilidade", cujos resultados parciais são apresentados neste dossiê. Parte do suporte provido pelo Instituto consistiu na realização de uma escola de inverno sobre metodologias transdisciplinares, em 2018, com grupos que centram sua atuação em pesquisas aplicadas, desenvolvidas junto com os interessados no problema em foco.

O desafio de se consolidar um instituto que contribua com a internacionalização da instituição, aprofundamento das pesquisas e novas metodologias de trabalho, incorporando práticas transdisciplinares, tem sido enfrentado pelas várias diretorias e demais participantes do IEAT ao longo dos anos, buscando sempre se ajustar às condições da UFMG como uma instituição pública que enfrenta as adversidades políticas e econômicas do País e, simultaneamente, apontar para novas direções.

Frick, à época presidente do Conselho Diretor do Instituto Freiburg de Estudos Avançados, Dose e Ertel discutem as especificidades de um instituto conectado a uma universidade no artigo Mapeando o mundo dos institutos de estudos avançados sediados em universidades (Frick, Dose, \& Ertel, 2011). Quanto à relação entre o IEA e a universidade, aventam primeiramente a hipótese de:

Universidades, impossibilitadas de transformar sua configuração institucional, poderão ficar tentadas a concentrar recursos financeiros e intelectuais nesses centros e usá-los como seu carro-chefe ou instituição de proa, desviando a atenção das deficiências do 'resto', a saber, as faculdades que a constituem (p. 26).

Frick, Dose, e Ertel (2011) apontam um outro caminho, mais positivo, que

Entende os UBIAS como laboratórios - um campo experimental e vicário no qual as Universidades contemplam qual direção devem seguir no futuro. Os UBIAS se tornariam então indicadores da necessidade de tais processos de reorientação - e podem eles próprios se tornar uma ferramenta útil para conduzir essa mudança (p. 27).

Para o IEAT, a função de apontar caminhos é um desafio permanente. Trabalhando em sintonia com as diversas instâncias da UFMG, o IEAT tem, entre outras iniciativas: 

a) participado da elaboração de seu Plano de Desenvolvimento Institucio- nal;
b) auxiliado iniciativas que não encontram apoio na estrutura departamen- tal, tal como a articulação de propostas de programas de pós-graduação interdisciplinares (no caso, em Estudos Brasileiros, Cidades e Bioenge- nharia);
c) buscado tornar o processo de ingresso na carreira docente menos corpora- tivo;
d) dado suporte para eventos e projetos interdisciplinares propostos por pes- quisadores;
e) oferecido disciplinas abertas para a graduação e pós-graduação;
f) organizado seminários sobre temas da atualidade e de reflexo na comuni- dade acadêmica.

A relação do Instituto com os alunos e o ensino de graduação, ao contrário de outros IEA, tem sido permanente. Ainda em 2003, o IEAT organizou uma disciplina com o tema Cidades, cujo objetivo foi construir metodologias transdisciplinares a partir de experiências inter e multidisciplinares. Professores e alunos de diversas áreas, estes últimos da graduação e pós-graduação, dedicaramse a atividades de campo e teóricas, constituindo uma experiência marcante para os participantes. Novas disciplinas em temas transdisciplinares ocorreram a partir de 2016. Em 2011, os seminários intitulados $A$ universidade do futuro, promovidos pelo Reitorado da UFMG em parceria com o IEAT, buscaram "Discutir o futuro levando em conta experiências, situações e perspectivas diversas e significativas das Américas, da Europa, das Asiáticas" (Institutos de Estudos Avançados Transdisciplinares, 2015). Posteriormente, em 2015, o Colóquio educação superior: dimensões e perspectivas transdisciplinares teve por objetivo induzir a criação de ambientes e mecanismos que favoreçam o entrelaçamento do conhecimento e práticas, abrangendo as diversas áreas para a formação profissional, com a proposta de subsidiar reformas e melhorias no ensino superior.

Uma parte comum e central aos IEA estrangeiros, qual seja, a presença de fellows que permanecem por períodos mais longos nos institutos, é especialmente difícil em países submetidos a instabilidades e orçamentos reduzidos para ciência, tecnologia e educação. Como exemplo, nos últimos quatro anos no Brasil, os cortes em recursos para a área da Educação foram dramáticos e de consequências duradouras. A busca de fontes alternativas para sustentar programas de cátedras é uma constante. O IEAT, felizmente, tem um fundo permanente que hoje ultrapassa um milhão de dólares e cujos rendimentos financiam, desde 2003, 
a vinda de catedráticos à universidade, graças à visão e empenho do professor Francisco César de Sá Barreto, reitor da UFMG de 1998 a 2002 e idealizador do Instituto.

Quanto à permanência de fellows por maiores períodos, o Programa Residente, existente no IEAT, permite a professores da UFMG passarem um período de 12 meses desenvolvendo um projeto de pesquisa, estando dispensados de suas atividades didáticas e administrativas. Assim se consegue um grupo que interage de forma sistemática, participa e auxilia nas atividades do Instituto e, idealmente, cria vínculos com o IEAT para futuras atividades. Similarmente, os grupos de pesquisa abrigados pelo IEAT são apoiados pelo Instituto em sua fase de estruturação, permanecendo vinculados por dois anos. Espera-se que, findo esse período, os grupos estejam maduros para se desenvolverem autonomamente. Como esse núcleo de residentes e grupos de pesquisa está em constante renovação, evita-se o risco de cristalização de grupos no Instituto a longo prazo.

As interações com outros IEA e organismos, no Brasil e exterior, são também ponto central para o Instituto. Em conjunto com outros institutos de estudos avançados no País, a UFMG hospedou, em 2015, a III Reunião de Institutos de Estudos Avançados brasileiros, na qual foi fundado o Fórum Brasileiro de Estudos Avançados (Fobreav), visando a propor e executar iniciativas e programas para a integração entre universidades, governo, empresas e organizações sociais, integrando redes de conhecimento baseadas em práticas inter e transdisciplinares. O Fórum considera a transversalidade como fundamental para a produção da ciência, da cultura e das tecnologias, a fim de incrementar o desenvolvimento científico, socioeconômico e cultural do País. Durante essa reunião de 2015, os representantes dos IEA brasileiros elaboraram um manifesto, descrito na Carta de Belo Horizonte (Institutos de Estudos Avançados Transdisciplinares, 2015).

O IEAT participa da rede Maria Sibylla Merian Center for Advanced Latin American Studies in the Humanities and Social Sciences (CALAS), composta de universidades latino-americanas e alemãs. Uma cátedra da CALAS está para ser implementada em breve.

Finalmente, o IEAT dispóe, junto à Editora UFMG, de uma coleção de livros dedicada a produções geradas ou diretamente relacionadas ao IEAT. São 16 títulos em papel e 4 com suporte digital. Participamos também da Revista da UFMG, de caráter interdisciplinar.

Em uma reunião de imersão realizada na cidade de Tiradentes, no fim de 2018, o IEAT definiu, por ocasião de seu vigésimo aniversário, seu objetivo: "Auxiliar a Universidade a se transformar em um ambiente propício à realização 
de estudos e resolução de problemas transdisciplinares". 3 Dessa reunião participaram os Conselhos Diretor e Científico, o corpo de funcionários, residentes, grupos de pesquisa e alguns poucos convidados da Reitoria e da Fundação de Desenvolvimento da Pesquisa (Fundep). As reflexões sobre a atualização do papel do IEAT após 20 anos, sua estratégia de comunicação, seus programas vigentes, vínculos com a estrutura da UFMG, seu papel no avanço de metodologias transdisciplinares na Universidade, elaboração de um plano de desenvolvimento institucional, função social do IEAT e internacionalização da UFMG são tópicos sendo trabalhados em comissões, formadas durante o evento em Tiradentes, para implantação nos próximos anos.

Em resumo, muitas foram as decisões que moldaram a estrutura e a prática do IEAT em seus primeiros 20 anos. De constante, uma disposição de permanentemente se renovar e trazer novas ideias e práticas avançadas para a UFMG. 


\section{REFERÊNCIAS}

Brewer, G. D. (1999). The challenges of interdisciplinarity. Policy Sciences, 32(4), 327-337.

Flexner, A. (1939). The usefulness of useless knowledge. Harpers Magazine, 139. Recuperado a partir de https://www.ias.edu/ideas/usefulness-uselessknowledge

Frick, W., Dose, C., Ertel, A. (2011). Mapeando o mundo dos institutos de estudos avançados sediados em universidades. C. Malferrari (Trad.). Estudos Avançados, 25(73), 19-30. Recuperado a partir de http://www.scielo.br/pdf/ ea/v25n73/a02v25n73.pdf

Goddard, P. (2011). O crescimento dos institutos de estudos avançados. C. Malferrari (Trad.). Estudos Avançados, 25(73), 7-18.

Institute for Advanced Study. (2019). The mission of the Institute for Advanced Study. Princeton: IAS. Recuperado a partir de https://www.ias.edu/ias-1-page

Instituto de Estudos Avançados Transdisciplinares. Institutos criam o Fórum Brasileiro de Estudos Avançados (2015). Belo Horizonte: Universidade Federal de Minas Gerais. Recuperado a partir de https://www.ufmg.br/ieat/2015/08/ institutos-criam-o-forum-brasileiro-de-estudos-avancados/

Netherlands Institute for Advanced Study. (2019). History. Amsterdã: NIAS. Recuperado a partir de https://nias.knaw.nl/who-we-are/history/

Pohl, C. \& Hadorn, G. H. (2007). Principles for designing transdisciplinary research: proposed by the Swiss Academies of Arts and Sciences. Munique: Oekon.

Resolução no 8 (24 junho, 1999). Cria, em caráter experimental, o Instituto de Estudos Avançados Transdisciplinares da UFMG (IEAT). Belo Horizonte: Conselho de Ensino, Pesquisa e Extensão da Universidade Federal de Minas Gerais.

Stokes, D. E. (1997). Pasteur's quadrant: basic science and technological innovation. Washington: Brokings Institution Press.

Van Breda, J. \& Swilling, M. (2018). The guiding logics and principles for designing emergent transdisciplinary research processes: learning experiences and reflections from a transdisciplinary urban case study in Enkanini informal settlement, South Africa. Sustainability Science, 14(3), 823-841. 
Wittrock, B. (2019). A brief history of institutes for advanced study. Oslo: Centre for Advanced Study, Norwegian Adacemy of Science and Letters. Recuperado a partir de https://cas.oslo.no/getfile.php/137422-1457965964/CAS_ publications/Jubilee\%20booklets/PDF/History_of_institutes.pdf 